\title{
Bimodal mesoporous silica with bottleneck pores
}

Michael J. Reber and Dominik Brühwiler*

Institute of Chemistry and Biological Chemistry

Zürich University of Applied Sciences

CH-8820 Wädenswil, Switzerland

* Corresponding author: dominik.bruehwiler@zhaw.ch

Dalton Transactions 44 (2015) 17960 - 17967. 


\section{Abstract}

Bimodal mesoporous silica consisting of two sets of well-defined mesopores is synthesized by a partial pseudomorphic transformation of an ordered mesoporous starting material (SBA-15 type). The introduction of a second set of smaller mesopores (MCM-41 type) establishes a pore system with bottlenecks that restricts the access to the core of the bimodal mesoporous silica particles. The particle size and shape of the starting material are retained, but micropores present in the starting material disappear during the transformation, leading to a true bimodal mesoporous product. A varying degree of transformation allows the adjustment of the pore volume contribution of the two mesopore domains. Information on the accessibility of the mesopores is obtained by the adsorption of fluorescence-labeled poly(amidoamine) dendrimers and imaging by confocal laser scanning microscopy. This information is correlated with nitrogen sorption data to provide insights regarding the spatial distribution of the two mesopore domains. The bimodal mesoporous materials are excellent model systems for the investigation of cavitation effects in nitrogen desorption isotherms. 


\section{Introduction}

Since their discovery in the early $1990 \mathrm{~s}^{1,2}$ ordered mesoporous silica based materials have had a substantial impact on research in various fields, including catalysis, ${ }^{3-6}$ separation, $^{6-8}$ drug delivery, ${ }^{9-11}$ sensing, ${ }^{9,12,13}$ and environmental remediation. ${ }^{14-16}$ A typical mesoporous silica material features a large surface area (reaching values of up to $1000 \mathrm{~m}^{2} \mathrm{~g}^{-1}$ ), a well-defined pore size (often in the range of 3 to $20 \mathrm{~nm}$ ), as well as abundant surface silanol groups, which allow postsynthetic functionalization and thus adjustment of the surface properties. ${ }^{17,18}$ Research on bimodal porous silica materials has focused on combining the beneficial properties of macro- and mesopores, e.g. fast mass transport (macropores) and large surface area (mesopores). ${ }^{19}$ Micro-mesoporous materials are similarly well-known, and can for example be generated by templating with nonionic triblock copolymers consisting of a central hydrophobic poly(propylene oxide) chain flanked by two hydrophilic poly(ethylene oxide) chains. ${ }^{20}$ It has been observed that the well-defined mesopores in these materials are interconnected by disordered micropores and smaller mesopores. ${ }^{21,22}$ Pathways to micro-mesoporous materials also include destructive synthesis strategies. These approaches generally start with zeolite crystals (microporous aluminosilicates) and introduce the mesopores by selectively extracting zeolite framework atoms. ${ }^{23}$ Constructive strategies towards micro-mesoporous materials with zeolitic domains typically involve the use of multiple templates or the assembly of zeolite-based building blocks. ${ }^{23}$

Bimodal materials with two types of mesopores are comparatively less well studied. These have been synthesized by mixing multiple templates to create a three-dimensional network of pores. ${ }^{24,25}$ Crosslinking of mesoporous MCM-41 nanoparticles in the presence of triblock copolymer surfactants is a further possibility to generate bimodal mesoporous silica. ${ }^{26}$ While these methods are successful at producing bimodal pore size distributions in the mesopore range, the degree of control over the particle size and shape, as well as over the spatial distribution of the two mesopore domains is limited. 
The generation of a mesoporous shell around a solid core silica particle is an excellent way of controlling the spatial distribution of the pore system within the final particle, but does not allow for a true bimodal character. ${ }^{27,28}$ Using a concept derived from pseudomorphic transformation, we were able to synthesize materials with two well-defined mesopore domains. The controlled dissolution and reprecipitation of silica during pseudomorphic transformation in the presence of a structure-directing agent ensures the retention of the initial particle size and shape. ${ }^{29}$ Starting with mesoporous silica and subjecting it to partial pseudomorphic transformation in the presence of a suitable structure-directing agent generates a bimodal material. The particle size and shape are defined by the starting mesoporous silica, whereas the two distinct pore sizes are determined by the structure-directing agents used in the synthesis of the starting mesoporous silica and in the subsequent pseudomorphic transformation. The relative contributions of the two mesoporous domains to the total pore volume of the final bimodal material can be adjusted by controlling the degree of pseudomorphic transformation.

Confocal laser scanning microscopy (CLSM) has previously been used to investigate the distribution of functional groups in mesoporous silica and to assess the accessibility of mesopores. ${ }^{30-32}$ We have applied this concept to the bimodal mesoporous materials in order to investigate the accessibility of the primary mesopores (provided by the starting material) after the introduction of smaller mesopores. The information on accessibility obtained by CLSM can be correlated with nitrogen sorption data to provide insights regarding the spatial distribution of the mesopore domains. 


\section{Experimental section}

Materials. Pluronic P123 $\left(\mathrm{EO}_{20} \mathrm{PO}_{70} \mathrm{EO}_{20}, \mathrm{M}_{\mathrm{n}} \approx 5800 \mathrm{~g} \mathrm{~mol}^{-1}\right)$, tetraethyl orthosilicate (TEOS, $\left.\geq 99 \%\right)$, sodium hydroxide (97\%), aqueous ammonia (25\%), hydrochloric acid (32\%), fluorescein isothiocyanate isomer 1 (FITC, $\geq 90 \%$ ), and poly(amidoamine) dendrimers generation 1 and 3 (PAMAM G1, PAMAM G3, both 20 wt\% in methanol) were obtained from Sigma Aldrich. Hexadecyltrimethylammonium bromide (CTAB, 99+\%) was obtained from Acros. Methanol (reagent grade) was obtained from Honeywell. Dodecyltrimethylammonium bromide (DTAB, $\geq 98 \%)$ and ethanol $(99.8 \%)$ were obtained from Fluka. Kromasil silica spheres $(5 \mu \mathrm{m}, 30 \mathrm{~nm})$ were obtained from AkzoNobel. All chemicals were used as received.

Synthesis of SBA-15. Following a previously published procedure, ${ }^{20} 2.2 \mathrm{~g}$ of P123 was dissolved in $80 \mathrm{~mL}$ of $1.55 \mathrm{M}$ hydrochloric acid. Once a clear solution was obtained, $5 \mathrm{~mL}$ of TEOS was slowly added. The mixture was stirred for $20 \mathrm{~h}$ at $35^{\circ} \mathrm{C}$, transferred to a Teflon-lined autoclave, and placed in a preheated oven at $100{ }^{\circ} \mathrm{C}$ for $24 \mathrm{~h}$. The autoclave was allowed to cool to room temperature before the product was recovered by filtration and washing with at least $1 \mathrm{~L}$ of $\mathrm{H}_{2} \mathrm{O}$. The material was allowed to dry at room temperature overnight and then calcined in air at $500{ }^{\circ} \mathrm{C}$ for $16 \mathrm{~h}$. A heating rate of $1{ }^{\circ} \mathrm{C} \min ^{-1}$ was applied.

Synthesis of MCM-41. Following a previously published procedure, ${ }^{33} 2.2 \mathrm{~g}$ of CTAB was dissolved in a solution of $52 \mathrm{~mL}$ of $\mathrm{H}_{2} \mathrm{O}$ and $25 \mathrm{~mL}$ of aqueous ammonia (25\%). After dropwise addition of $10 \mathrm{~mL}$ of TEOS, the mixture was stirred at room temperature for $3 \mathrm{~h}$. It was then transferred to a Teflon-lined autoclave and put in an oven at $110^{\circ} \mathrm{C}$ for $48 \mathrm{~h}$. The autoclave was allowed to cool to room temperature before the product was recovered by filtration and washing with at least $250 \mathrm{~mL}$ of $\mathrm{H}_{2} \mathrm{O}$. The material was allowed to dry at room temperature overnight. The structure-directing agent was removed by heating at $300^{\circ} \mathrm{C}$ for $2 \mathrm{~h}$ and subsequent calcination at $500{ }^{\circ} \mathrm{C}$ for $12 \mathrm{~h}$. A heating rate of $1^{\circ} \mathrm{C} \min ^{-1}$ was applied. 
Synthesis of SBA-s. Spherical particles of SBA-15 (SBA-s) were synthesized as follows: ${ }^{30}$ An amount of $465 \mathrm{mg}$ of CTAB was dissolved in $20 \mathrm{~mL}$ of $\mathrm{H}_{2} \mathrm{O}$. This solution was added to a solution of $3.1 \mathrm{~g}$ of P123 in $46 \mathrm{~mL}$ of $1.5 \mathrm{M}$ hydrochloric acid, and $7.8 \mathrm{~mL}$ of ethanol was added, followed by the dropwise addition of $10 \mathrm{~mL}$ of TEOS. The mixture was stirred for $2 \mathrm{~h}$ at room temperature before being transferred to a Teflon-lined autoclave. The autoclave was placed in a preheated oven at $78{ }^{\circ} \mathrm{C}$ for $72 \mathrm{~h}$. The autoclave was allowed to cool to room temperature and the product was recovered by filtration and washing with at least $1 \mathrm{~L}$ of $\mathrm{H}_{2} \mathrm{O}$. The material was allowed to dry at room temperature overnight and then calcined in air at $500{ }^{\circ} \mathrm{C}$ for $16 \mathrm{~h}$. A heating rate of $1{ }^{\circ} \mathrm{C} \mathrm{min}^{-1}$ was applied.

Pseudomorphic transformation. An amount of $300 \mathrm{mg}$ of the parent silica (SBA-15, SBA-s or Kromasil) was mixed with $182 \mathrm{mg}$ of CTAB and a varying amount of $\mathrm{NaOH}$. The ratio of the mass of silica $\left(m_{\text {silica }}\right)$ and $\mathrm{NaOH}\left(m_{\mathrm{NaOH}}\right)$ for each synthesis is given in Table 1. To this mixture, $2 \mathrm{~mL}$ of $\mathrm{H}_{2} \mathrm{O}$ was added and stirred for $30 \mathrm{~min}$ before being transferred to a Teflon-lined autoclave. The autoclave was placed in an oven at $100{ }^{\circ} \mathrm{C}$ for $6 \mathrm{~h}$ in the case of SBA-15 or $24 \mathrm{~h}$ in the case of SBA-s and Kromasil. The autoclave was allowed to cool to room temperature. The product was filtered, washed with $250 \mathrm{~mL}$ of $\mathrm{H}_{2} \mathrm{O}$, and calcined in air at $550{ }^{\circ} \mathrm{C}$ for $12 \mathrm{~h}$. A heating rate of $1{ }^{\circ} \mathrm{C} \mathrm{min}-1$ was applied. For samples made with DTAB instead of CTAB, $154 \mathrm{mg}$ of the structure-directing agent was used.

Accessibility tests. FITC coupled PAMAM G1 and G3 were used as fluorescent probes for the accessibility tests. The stock solutions were made by taking $1 \mathrm{~mL}$ of PAMAM solution ( $20 \mathrm{wt} \%$ in methanol) and adding FITC. The amount of added FITC was calculated to give one FITC molecule for every 16 primary amino groups. The solution was diluted with $5 \mathrm{~mL}$ of methanol and stirred for $24 \mathrm{~h}$. An amount of $500 \mu \mathrm{L}$ of this solution was added to $50 \mathrm{mg}$ of silica and stirred for $8 \mathrm{~h}$. The solvent was then allowed to evaporate. Before CLSM images were taken, the PAMAM-loaded silica was placed in an oven at $80^{\circ} \mathrm{C}$ for $1 \mathrm{~h}$. 
Physical measurements. Nitrogen sorption isotherms were measured at $77 \mathrm{~K}$ with a Quantachrome Autosorb iQ MP. Samples were vacuum-degassed for $3 \mathrm{~h}$ at $80{ }^{\circ} \mathrm{C}$ before measurement. Pore size distributions and pore diameters $d_{\mathrm{DFT}}$ were determined by a nonlocal density functional theory (NLDFT) model developed for silica exhibiting cylindrical pore geometry. ${ }^{34}$ Surface areas $S_{\mathrm{BET}}$ were calculated with a multipoint BET method. ${ }^{35}$ Total pore volumes $V_{\text {tot }}$ were derived from the amount of adsorbed nitrogen at a relative pressure of $\mathrm{p} / \mathrm{p}_{0}=0.99$. The de Boer equation was used for calculating the statistical film thickness (t-plot). ${ }^{36}$ Adsorption isotherms were used for all calculations. Scanning electron microscopy (SEM) images were taken with a FEI Quanta FEG 250. CLSM images were acquired with an Olympus BX60 microscope equipped with a FluoView FV300 confocal unit and excitation at $488 \mathrm{~nm}$.

\section{Results and discussion}

Nomenclature. Materials prepared by pseudomorphic transformation of SBA-15 are designated as Sq-x, where $q$ indicates the alkyl chain length of the structure-directing agent $(q=16$ for CTAB, $q=12$ for DTAB) and $x$ represents the degree of pseudomorphic transformation. This value corresponds to the percentage of pore volume that is contributed by the MCM-41 domains introduced during pseudomorphic transformation. Materials synthesized from SBA-s (spherical SBA-15) or Kromasil are denoted as S-Sq-x and K-Sq-x, respectively. The samples along with the key structural data obtained from nitrogen sorption are summarized in Table 1. 
Table 1. Structural data of parent and transformed materials.

\begin{tabular}{lcccc}
\hline Sample & $d_{\mathrm{DFT}} / \mathrm{nm}$ & $S_{\mathrm{BET}} / \mathrm{m}^{2} \mathrm{~g}^{-1}$ & $V_{\text {tot }} / \mathrm{cm}^{3} \mathrm{~g}^{-1}$ & $m_{\text {silica }} / m_{\mathrm{NaOH}}$ \\
\hline SBA-15 & 7.3 & 570 & 0.84 & - \\
SBA-S & 5.9 & 745 & 0.71 & - \\
MCM-41 & 3.7 & 670 & 0.63 & - \\
S16-100 & 3.9 & 661 & 0.54 & 6 \\
S16-84 & $4.1,6.5$ & 623 & 0.61 & 12 \\
S16-23 & $4.0,6.6$ & 536 & 0.70 & 15 \\
S12-46 & $3.2,6.5$ & 627 & 0.63 & 12 \\
S-S16-99 & 3.5 & 544 & 0.45 & 6 \\
S-S16-55 & $4.2,5.6$ & 556 & 0.56 & 15 \\
K-S16-100 & 3.7 & 587 & 0.56 & 6 \\
\hline
\end{tabular}

Pseudomorphic transformation of SBA-15. The pseudomorphic transformation pathway is able to take a parent porous material and convert its typically disordered macroporous structure to a framework with an ordered pore system and a well-defined pore size. ${ }^{29}$ The particle size and shape of the parent material are retained during the process. We have used SBA-15, which features a narrow pore size distribution centered at $7.3 \mathrm{~nm}$, as a starting material (Figure 1a). SBA-15 contains micropores and small mesopores that provide connectivity between the large primary mesopores. ${ }^{21,37}$ Through pseudomorphic transformation of SBA-15 in the presence of $\mathrm{CTAB}$, it is possible to convert the material completely to a MCM-41 type material (sample S16-100). The pore condensation step in the nitrogen adsorption isotherm shifts to lower relative pressure and the pore size is reduced from 7.3 to $3.9 \mathrm{~nm}$ (Figure 1c). The BET surface area remains high and even increases slightly (Table 1). However, it should be noted that the value of the BET surface area for SBA-15 needs to be interpreted with caution due to the presence of 
micropores. ${ }^{38}$ As will be shown in the following, these micropores disappear upon pseudomorphic transformation.

In terms of the structural properties, the resulting sample S16-100 is very similar to MCM-41 prepared by classical liquid crystal templating (Figure 1b), although the pore size distribution of the classical MCM-41 is slightly narrower and shifted to smaller pore size due to the different reaction conditions. No significant effect of the pseudomorphic transformation on the particle size and shape could be observed (Figure 2).
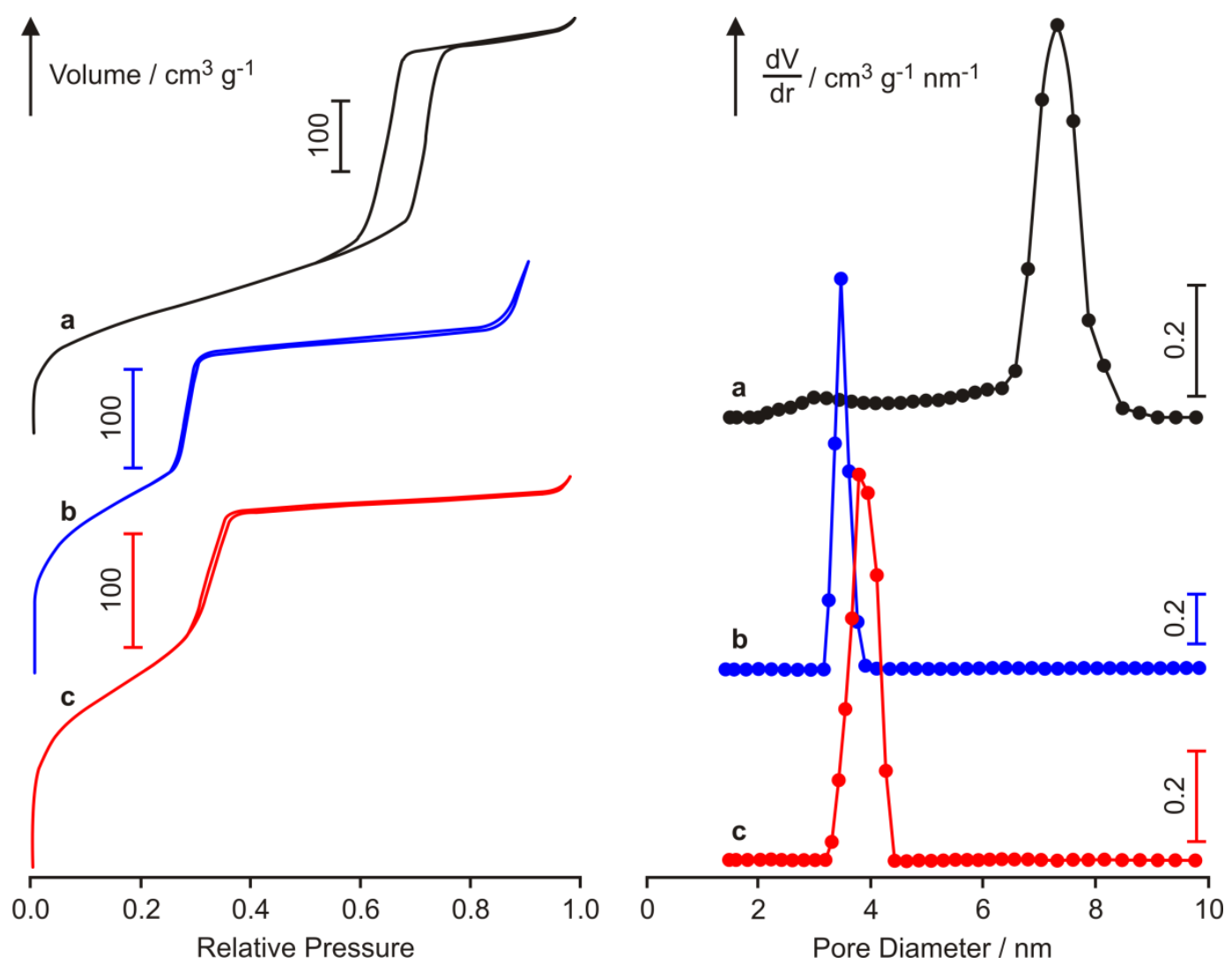

Figure 1. Left: Nitrogen sorption isotherms of SBA-15 (a), MCM-41 (b), and S16-100 (c). The corresponding pore size distributions are shown on the right. The isotherms and pore size distributions are offset for clarity. 

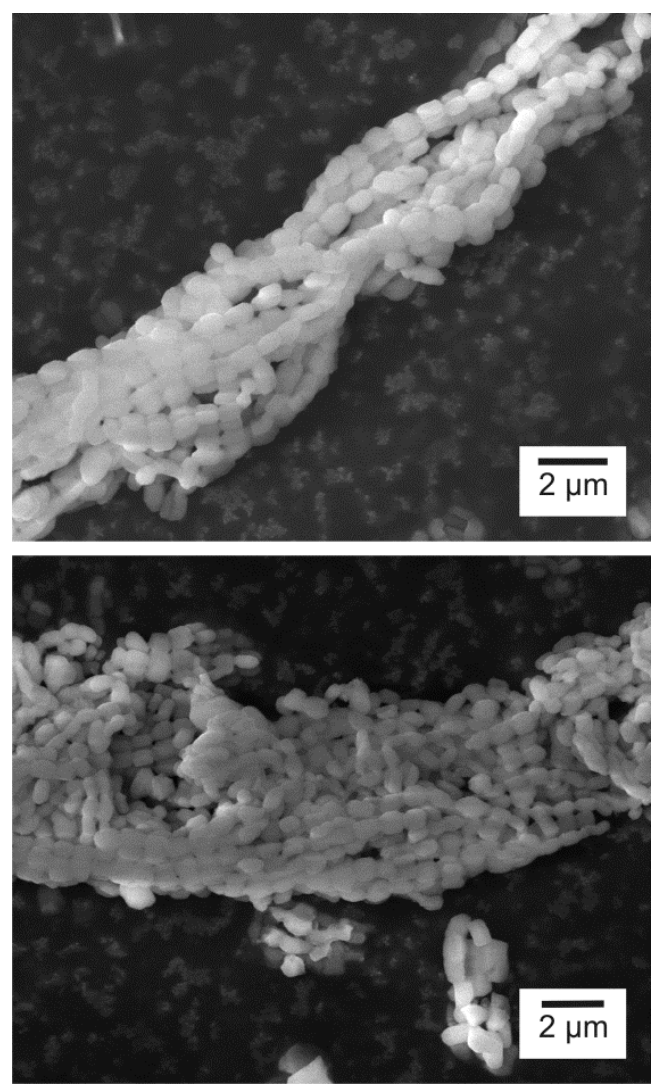

Figure 2. SEM images of parent SBA-15 (top) and the fully transformed sample S16-100 (bottom).

Interestingly, the micropores and small secondary mesopores of the parent SBA-15 disappear during the pseudomorphic transformation to S16-100. Comparison of the $t$-plots of the materials provides additional evidence for the elimination of the micropores during pseudomorphic transformation. At low $t$-values, a pronounced deviation from linearity can be observed for the parent SBA-15, whereas the product of the transformation is characterized by a more linear $t$-plot in the same range (Figure 3 ), comparable to MCM-41. The high $\mathrm{pH}$ of the pseudomorphic transformation apparently fuses the micropores of SBA-15. 


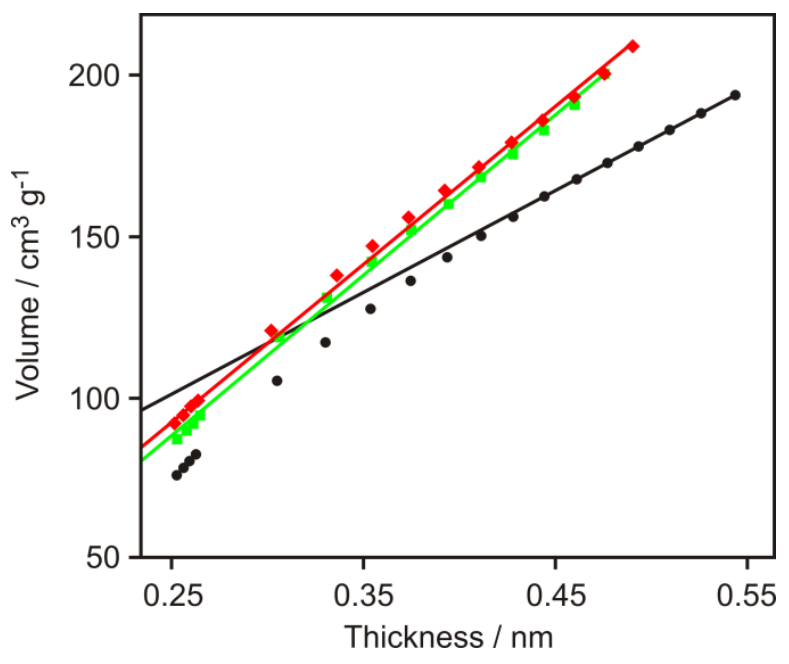

Figure 3. $t$-Plots of parent SBA-15 (black, circles), MCM-41 (red, diamonds), and S16-100 (green, rectangles).

Bimodal materials derived from SBA-15. Reducing the concentration of $\mathrm{NaOH}$ in the pseudomorphic transformation of SBA-15 yields bimodal mesoporous silica. This partial transformation leads to materials that contain both SBA-15 and MCM-41 type domains. Nitrogen adsorption isotherms of these bimodal materials show two distinct pore condensation steps (Figure 4). The first step is located at $p / p_{0}=0.36$ $(\mathrm{MCM}-41)$ and the second at $\mathrm{p} / \mathrm{p}_{0}=0.72(\mathrm{SBA}-15)$. The two distinct domains can be observed in the corresponding pore size distributions. The amount of the transformation and thus the relative contribution of the two domains to the total pore volume can be changed by adjusting the amount of $\mathrm{NaOH}$ in the reaction mixture, thereby creating a sliding scale of SBA-15 and MCM-41 domains in the transformed material (Table 1, Figure 4). 

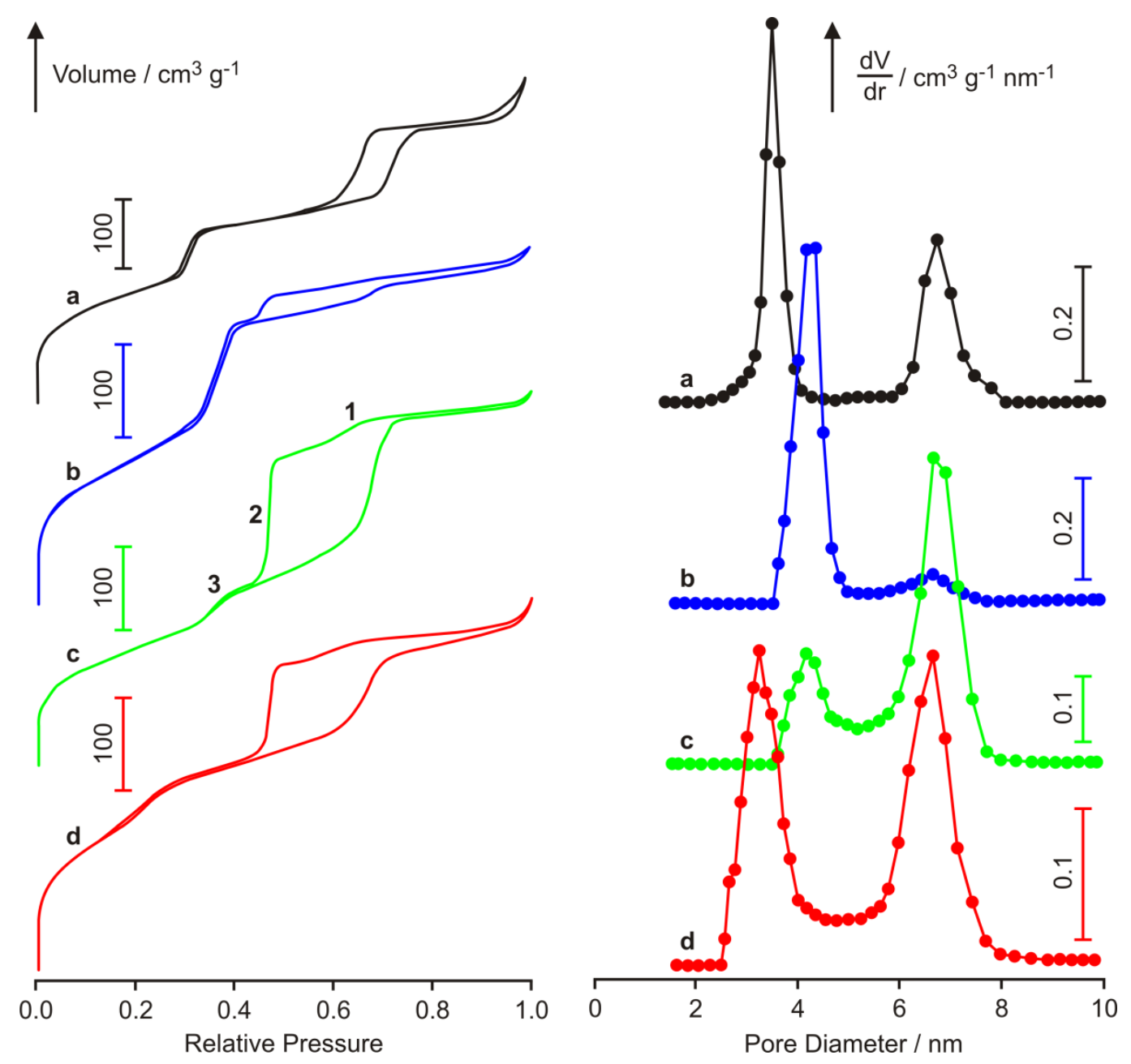

Figure 4. Left: Nitrogen sorption isotherms of a mechanical mixture of SBA-15 and MCM-41 (a), S16-84 (b), S16-23 (c), and S12-46 (d). The corresponding pore size distributions are shown on the right. The isotherms and pore size distributions are offset for clarity.

SBA-15 and MCM-41 generally show type IV nitrogen sorption isotherms. MCM-41 tends to have a reversible isotherm, while SBA-15 displays a $\mathrm{H} 1$ hysteresis due to delayed condensation. The delayed vapor-liquid transition is due to the existence of metastable fluid states. These metastabilities only exist in the adsorption isotherm, whereas the desorption proceeds through a receding meniscus and is considered the equilibrium liquid-vapor transition. ${ }^{39}$ The interpretation of the isotherms of hierarchically 
structured materials is more complex. The presence of cage-like structures allows for a different type of desorption. Desorption is delayed when adsorbed nitrogen must pass through a bottleneck. Percolation (pore blocking) and cavitation lead to $\mathrm{H} 2$ hysteresis, which is defined by a pronounced step in the desorption isotherm. The neck diameter determines which type of desorption mechanism is to occur. Above a critical diameter (ca. $5 \mathrm{~nm}$ for nitrogen at $77 \mathrm{~K}$ ), pore blocking delays desorption, whereas cavitation is involved below the critical diameter. ${ }^{40}$ During cavitation, gas bubbles nucleate and grow in the metastable condensed fluid. Desorption from the pore body occurs while the pore neck remains filled (or partially filled). At relative pressures between 0.42 and 0.50 (for nitrogen), spontaneous evaporation of the stretched metastable liquid occurs, causing a forced closure of the hysteresis loop. This phenomenon is often referred to as the tensile strength effect. ${ }^{41}$

All of the bimodal materials that we have obtained by partial pseudomorphic transformation of SBA-15 are characterized by a large $\mathrm{H} 2$ hysteresis. The adsorption isotherms feature two well separated steps, corresponding to condensation in the MCM-41 (low pressure) and in the SBA-15 domains (high pressure). The desorption isotherms are more complex. A mechanical mixture of MCM-41 and SBA-15 displays the expected reversible condensation for MCM-41 and the H1 hysteresis for SBA-15 (Figure 4a). The bimodal materials, however, give rise to a pronounced $\mathrm{H} 2$ hysteresis with three steps in the desorption branch (indicated by numbers in Figure 4c). The high pressure step 1 is comparable to the step in the $\mathrm{H} 1$ hysteresis of the parent SBA-15 and therefore most likely corresponds to desorption from unobstructed SBA-15 pores. Step 2 is extremely steep with a forced closure at $p / p_{0}=0.42$. This is a clear indication of the cavitation mechanism for pore emptying and points to SBA-15 domains that are only accessible through the smaller pores of the MCM- 41 domains. The pores of the MCM- 41 domains act as bottlenecks and desorption from these pores occurs in the typical reversible step 3.

The pore size of the MCM-41 domains can be adjusted by employing structure-directing agents with differently sized alkyl chains. Using DTAB in the pseudomorphic transformation (instead of CTAB) 
introduces MCM-41 domains with a pore size of $3.2 \mathrm{~nm}$ (Figure 4d). The forced closure of the desorption isotherm remains at $p / p_{0}=0.42$, confirming that the cavitation mechanism is independent of the neck diameter once this value is below the critical diameter. ${ }^{42}$

Accessibility tests. From the nitrogen sorption analysis we can conclude that the MCM-41 domains act as bottlenecks and therefore should restrict the access to a large part of the SBA-15 domains. Further experimental evidence for the structure of the partially transformed materials is provided by investigating the accessibility to the SBA-15 domains. PAMAM dendrimers coupled with FITC were used as probe molecules. PAMAM G1 (1.9 nm in diameter) is expected to be able to pass the MCM-41 domains and therefore access the entire particle, whereas $\mathrm{G} 3(3.6 \mathrm{~nm}$ in diameter) is too large to efficiently diffuse through the MCM-41 domains. ${ }^{43}$ Imaging the particles with CLSM allows us to analyze the spatial distribution of the adsorbed FITC-coupled PAMAM dendrimers. The applicability of this method depends on the size of the bimodal mesoporous silica particles. Previous accessibility studies and the analysis of functional group distributions by means of CLSM have used mesoporous silica particles in the size regime of $5 \mu \mathrm{m} .{ }^{30,32}$ We have therefore applied the partial pseudomorphic transformation to spherical SBA-15 particles (SBA-s) with a size in the range of 4 to $7 \mu \mathrm{m}$. The pore size distribution of SBA-s is shown in Figure 5. The synthetic conditions for SBA-s include ethanol and CTAB (as a co-surfactant), which promote the formation of spherical particles but also add disorder to the pore system, leading to a broader pore size distribution compared to the classical SBA-15 discussed above. It should also be noted that SBA-s contains a significant amount of secondary mesopores that have a smaller diameter than the primary mesopores. As can be seen from the pore size distribution of the transformed materials (Figure 5), the conditions of the pseudomorphic transformation lead to the elimination of these secondary mesopores.

After adsorption of FITC-coupled PAMAM (G1 or G3), optical slices in the center of the parent SBA-s, partially transformed (S-S16-55), and fully transformed (S-S16-99) particles were imaged by CLSM 
(Figure 5). The parent SBA-s particles are completely accessible to both G1 and G3. The small secondary mesopores apparently do not obstruct access to the particle core. Partial transformation of SBA-s leads to a bimodal material that is completely accessible to G1 but not to G3. The same is observed for the fully transformed material. In agreement with the nitrogen sorption analysis, this indicates that the MCM-41 domains act as bottlenecks and reduce the accessibility to the larger pores of the SBA-15 domains. Furthermore, the data suggest the presence of MCM-41 domains close to the external particle surface.
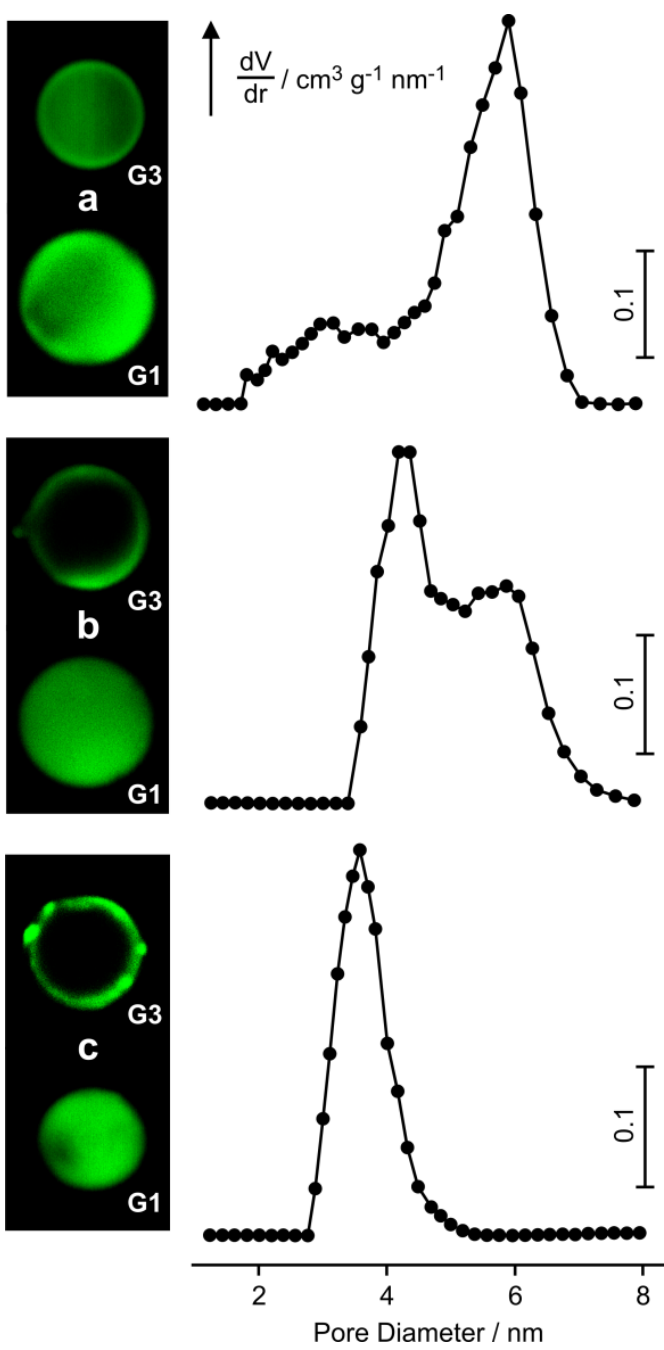

Figure 5. Left: CLSM images of PAMAM G3 and G1 adsorbed on SBA-s (a), S-S16-55 (b), and S-S16-99 (c). Right: Corresponding pore size distributions before PAMAM adsorption. 
In order to confirm that the MCM-41 domains are the limiting factor for accessibility, pseudomorphically transformed spheres with a much larger initial pore size $(>30 \mathrm{~nm})$ were studied. The commercial silica gel Kromasil was used for this purpose. As we have shown previously, Kromasil can be transformed into a MCM-41 type material. ${ }^{44}$ However, a small fraction of disordered large meso- and macropores remains after the transformation. ${ }^{44}$ As can be seen from the adsorption of $\mathrm{G} 3$, these remaining interconnecting large pores provide full access to the particle core (Figure 6). This is in contrast to the materials prepared from SBA-15 and SBA-s.

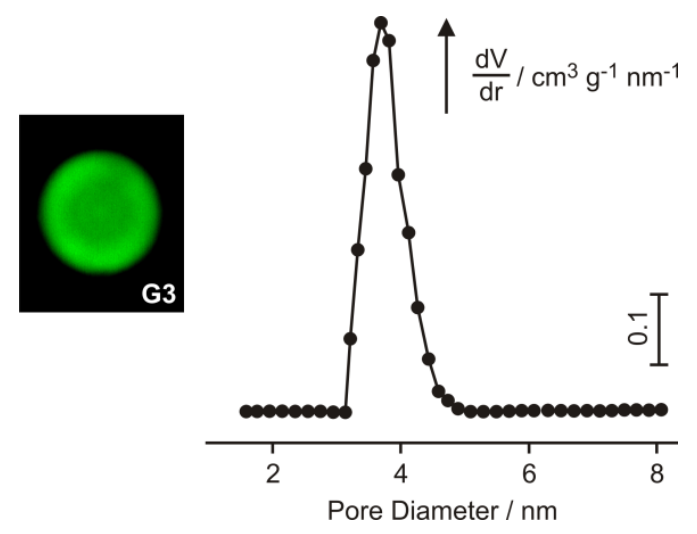

Figure 6. Left: CLSM image of PAMAM G3 adsorbed on K-S16-100. Right: Corresponding pore size distribution before PAMAM adsorption.

\section{Conclusions}

Partial pseudomorphic transformation of SBA-15 type materials with alkyltrimethylammonium structuredirecting agents yields bimodal mesoporous materials containing two sets of well-defined pore sizes. The smaller mesopores act as bottlenecks and restrict the access to the larger mesopores. Adsorption of fluorescence-labeled PAMAM dendrimers combined with CLSM imaging shows that the presence of these bottlenecks close to the external particle surface leads to size selectivity regarding the accessibility 
to the particle core. The diameter of the bottlenecks can be tuned by using alkyltrimethylammonium structure-directing agents with different alkyl chain lengths. The size of the larger mesopores, as well as the particle size and shape are determined by the starting material employed in the pseudomorphic transformation. Due to this high level of control over the structural parameters, the materials are ideal model systems for gas sorption studies and the elucidation of mechanisms underlying adsorptiondesorption hysteresis. From a preparative point of view, the bimodal mesoporous silicas are of interest as host materials for "ship-in-a-bottle" syntheses and for controlled release of guests by means of pore entrance (bottleneck) grafted gates. ${ }^{9,45-47}$

\section{Acknowledgements}

Financial support by the Swiss National Science Foundation (project 200021_140303) is acknowledged. 


\section{References}

1 J. S. Beck, J. C. Vartuli, W. J. Roth, M. E. Leonowicz, C. T. Kresge, K. D. Schmitt, C. T.-W. Chu, D. H. Olson, E. W. Sheppard, S. B. McCullen, J. B. Higgins and J. L. Schlenker, J. Am. Chem. Soc., 1992, 114, 10834-10843.

2 T. Yanagisawa, T. Shimizu, K. Kuroda and C. Kato, Bull. Chem. Soc. Jpn., 1990, 63, 988-992.

3 S. Rostamnia and E. Doustkhah, RSC Adv., 2014, 4, 28238-28248.

4 X. Qian, K. Fuku, Y. Kuwahara, T. Kamegawa, K. Mori and H. Yamashita, ChemSusChem, 2014, 7, 1528-1536.

5 Y. Lin, J. Ren and X. Qu, Acc. Chem. Res., 2014, 47, 1097-1105.

6 P. Van Der Voort, D. Esquivel, E. De Canck, F. Goethals, I. Van Driessche and F. J. Romero-Salguero, Chem. Soc. Rev., 2013, 42, 3913-3955.

7 I. Sierra, D. Pérez-Quintanilla, S. Morante and J. Gañán, J. Chromatogr. A, 2014, 1363, 27-40.

8 A. Ahmed, P. Myers and H. Zhang, Langmuir, 2014, 30, 12190-12199.

9 S. Alberti, G. J. A. A. Soler-Illia and O. Azzaroni, Chem. Commun., 2015, 51, 6050-6075.

10 N. Z. Knežević and J.-O. Durand, Nanoscale, 2015, 7, 2199-2209.

11 C. Argyo, V. Weiss, C. Bräuchle and T. Bein, Chem. Mater., 2014, 26, 435-451.

12 S. Widmer, M. J. Reber, P. Müller, C. E. Housecroft, E. C. Constable, R. M. Rossi, D. Brühwiler, L. J. Scherer and L. F. Boesel, Analyst, 2015, 140, 5324-5334.

13 T. Wagner, S. Haffer, C. Weinberger, D. Klaus and M. Tiemann, Chem. Soc. Rev., 2013, 42, 4036-4053.

14 Z. Zhang, H. Lan, H. Liu, H. Li and J. Qu, RSC Adv., 2015, 5, 42407-42413.

15 Y.-P. Zhu, T.-Z. Ren and Z.-Y. Yuan, RSC Adv., 2015, 5, 7628-7636.

16 H. Yoshitake, J. Mater. Chem., 2010, 20, 4537-4550.

17 D. Brühwiler, Nanoscale, 2010, 2, 887-892.

18 F. Hoffmann, M. Cornelius, J. Morell and M. Fröba, Angew. Chem., Int. Ed., 2006, 45, 3216-3251.

19 M. Antonietti, B. Berton, C. Göltner and H.-P. Hentze, Adv. Mater., 1998, 10, 154-159.

20 D. Zhao, Q. Huo, J. Feng, B. F. Chmelka and G. D. Stucky, J. Am. Chem. Soc., 1998, 120, 6024-6036.

21 R. Ryoo, C. H. Ko, M. Kruk, V. Antochshuk and M. Jaroniec, J. Phys. Chem. B, 2000, 104, 11465-11471.

22 M. Kruk, M. Jaroniec, C. H. Ko and R. Ryoo, Chem. Mater., 2000, 12, 1961-1968.

23 R. Chal, C. Gérardin, M. Bulut and S. van Donk, ChemCatChem, 2011, 3, 67-81.

24 R. Xing, H.-J. Lehmler, B. L. Knutson and S. E. Rankin, Langmuir, 2009, 25, 6486-6492.

25 O. Sel, D. Kuang, M. Thommes and B. Smarsly, Langmuir, 2006, 22, 2311-2322.

26 J. H. Sun, Z. Shan, T. Maschmeyer and M.-O. Coppens, Langmuir, 2003, 19, 8395-8402. 
27 S. B. Yoon, J.-Y. Kim, J. H. Kim, Y. J. Park, K. R. Yoon, S.-K. Park and J.-S. Yu, J. Mater. Chem., 2007, 17, $1758-1761$.

28 H. Dong and J. D. Brennan, J. Mater. Chem., 2012, 22, 13197-13203.

29 T. Martin, A. Galarneau, F. Di Renzo, F. Fajula and D. Plee, Angew. Chem., Int. Ed., 2002, 41, 2590-2592.

30 N. Gartmann and D. Brühwiler, Angew. Chem., Int. Ed., 2009, 48, 6354-6356.

31 K. Cheng and C. C. Landry, J. Am. Chem. Soc., 2007, 129, 9674-9685.

32 M. Ince, N. Gartmann, C. G. Claessens, T. Torres and D. Brühwiler, Org. Lett., 2011, 13, 4918-4921.

33 D. Brühwiler and H. Frei, J. Phys. Chem. B, 2003, 107, 8547-8556.

34 J. Landers, G. Y. Gor and A. V. Neimark, Colloid. Surf. A: Physicochem. Eng. Aspects, 2013, 437, 3-32.

35 S. Brunauer, P. H. Emmett and E. Teller, J. Am. Chem. Soc., 1938, 60, 309-319.

36 B. C. Lippens and J. H. de Boer, J. Catal., 1965, 4, 319-323.

37 P. I. Ravikovitch and A. V. Neimark, J. Phys. Chem. B, 2001, 105, 6817-6823.

38 A. Galarneau, H. Cambon, F. Di Renzo and F. Fajula, Langmuir, 2001, 17, 8328-8335.

39 P. A. Monson, Langmuir, 2008, 24, 12295-12302.

40 P. I. Ravikovitch and A. V. Neimark, Langmuir, 2002, 18, 9830-9837.

41 J. C. Groen, L. A. A. Peffer and J. Pérez-Ramírez, Microporous Mesoporous Mater., 2003, 60, 1-17.

42 P. T. M. Nguyen, D. D. Do and D. Nicholson, J. Phys. Chem. B, 2011, 115, 12160-12172.

43 R. Esfand and D. A. Tomalia, in Dendrimers and Other Dendritic Polymers, ed. J. M. J. Fréchet and D. A. Tomalia, John Wiley \& Sons, 2001, pp. 587-604.

44 M. J. Reber and D. Brühwiler, Part. Part. Syst. Charact., 2015, 32, 243-250.

45 Z.-A. Qiao, Q. Huo, M. Chi, G. M. Veith, A. J. Binder and S. A. Dai, Adv. Mater., 2012, 24, 6017-6021.

46 M. Shakeri, R. J. M. Klein Gebbink, P. E. de Jongh and K. P. de Jong, Angew. Chem., Int. Ed., 2013, 52, $10854-10857$.

47 A. Khorshidi and S. Heidari, RSC Adv., 2015, 5, 32804-32812. 